\title{
Differentiation of C-Positive Dots Across the Width of Chromosomes
}

\author{
Seiichi Sato \\ Biological Institute, Faculty of Science, \\ Ehime University, Matsuyama, Japan
}

Received April 7, 1979

Many different banding techniques, including C-banding, have exposed a linear differentiation of chromosomes along their length. However, in the chromosomes of Nothoscordum fragrans, some C-banding positive segments have been found to be composed of several dot-like compartments arranged linearly across the width of the chromosomes. The $\mathrm{C}$-banding positive segments of the chromosomes of this plant were characterized into three groups according to both the sites in which they appeared and their histochemical response to the fluorochromes (Sato 1980). The present work was performed in order to characterize the organization of the dotlike compartments among the three groups of $\mathrm{C}$-banding positive segments.

\section{Materials and methods}

The Nothoscordum fragrans used in the present observations was of the clone $\left(2 n=19=13 L^{m}+6 S^{a c}\right)$ preserved in the experimental garden of Ehime University. The root tips were excised from plantlets grown in pots and immediately fixed in a mixture of ethanol 3: glacial acetic acid 1 at $0-4^{\circ} \mathrm{C}$ for $1 \mathrm{hr}$ without pretreatment. The procedure for C-banding is described in a previous report (Sato et al. 1979). Details of the karyotype and the C-banding pattern of this plant have already been reported (Sato et al. ${ }^{a}$ 1980, Sato et al. 1980).

\section{Observations}

Fig. 1a shows the C-banding pattern in the metaphase chromosomes of Nothoscordum fragrans. As reported in the previous paper (Sato 1980), the C-banding positive segments of this plant can be classified into the following three groups: 1) I-segments (C-banding positive segments appearing on the interstitial regions of $\mathrm{S}^{\mathrm{ac}}$ chromosomes), 2) S-segments (those on the short arms of $\mathrm{S}^{\text {ac }}$ chromosomes), and 3) M-segments (those on the distal regions of $L^{m}$ chromosomes).

The I-segments sometimes appeared as a complex of dot-like compartments (C-positive dots), which were usually less than $1 \mu \mathrm{m}$ in diameter (Fig. 1b). As summarized in Table 1, the maximum number of C-positive dots per I-segment was four. As shown in Fig. 1b, they were not always arranged linearly across the width of the chromosomes. This phenomenon probably resulted from slipping of the sister chromatids when the chromosomes were squashed under the cover slips. 
In the metaphase chromosomes, the I-segments composed of three $\mathrm{C}$-positive dots were most frequently seen (Fig. 1c) followed by those composed of two. The Isegments appeared rarely as a single block with a diameter of about $1 \mu \mathrm{m}$. Usually, the size of the C-positive dots in the I-segments composed of only one or two dots

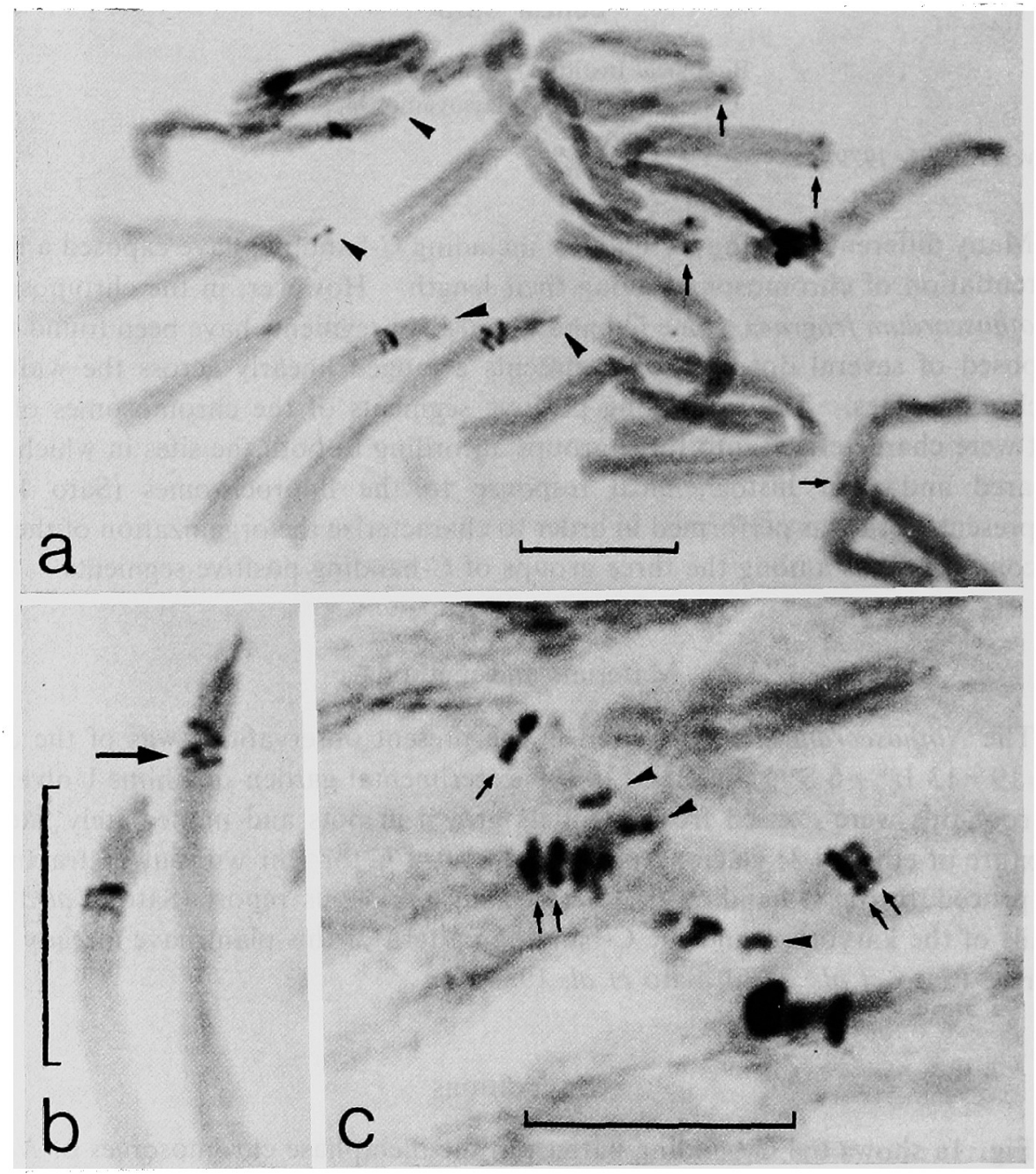

Fig. 1. Metaphase chromosomes revealed by the C-banding technique. a, a representative metaphase plate. The $\mathrm{C}$-banding positive segments are seen at the interstitial regions, the short arms (wedges) and the distal regions (arrows). b, enlargement of a part of Fig. 1a. Some I-segments are clearly found to be composed of four C-positive dots (arrow). c, in this view of C-banded metaphase chromosomes, the short arms appear as two C-positive dots (wedges), and the I-segments are composed of three or four C-positive dots which are arranged linearly across the width of the chromosomes (arrows). Bar $=10 \mu \mathrm{m}$.

was larger than that in the I-segments composed of three or four dots. $\mathrm{S}^{\mathrm{ac}}-3$ chromosomes with the large and the fine I-segments were excluded from the present observations since the large ones always appeared as blocks with a diameter of more than $1 \mu \mathrm{m}$, and the fine ones frequently disappeared during the $\mathrm{C}$-banding procedure 
(Fig. 1a). Almost all of the S-segments were composed of a pair of C-positive dots, and each was a short arm of sister chromatid (Fig. 1c). The M-segment was usually observed as a pair of C-positive dots (Fig. 1a). The size of the C-positive dots at an $\mathrm{M}$-segment was never as great as the width of a sister chromatid and they were situated on the edge of each sister chromatid. In almost all of the $\mathrm{L}^{\mathrm{m}}$ chromosomes

Table 1. Frequency distribution of the C-positive dots for the three types of $\mathrm{C}$-banding positive segments

\begin{tabular}{cccccc}
\hline \multirow{2}{*}{ Chromosome } & $\begin{array}{c}\text { Type of C-banding } \\
\text { positive segment }\end{array}$ & \multicolumn{5}{c}{ Number of C-positive dots per segment } \\
& I & 1 & 2 & 3 & 4 \\
\hline Prophase & S & 2 & 19 & 4 & 1 \\
& M & 0 & 15 & 0 & 0 \\
Metaphase & I & 2 & 23 & 49 & 0 \\
& S & 5 & 28 & 0 & 0 \\
Anaphase & M & 2 & 38 & 0 & 0 \\
& I & 32 & 128 & 0 & 0 \\
& S & 42 & 4 & 0 & 0 \\
& M & 55 & 0 & 0 & 0 \\
\hline
\end{tabular}

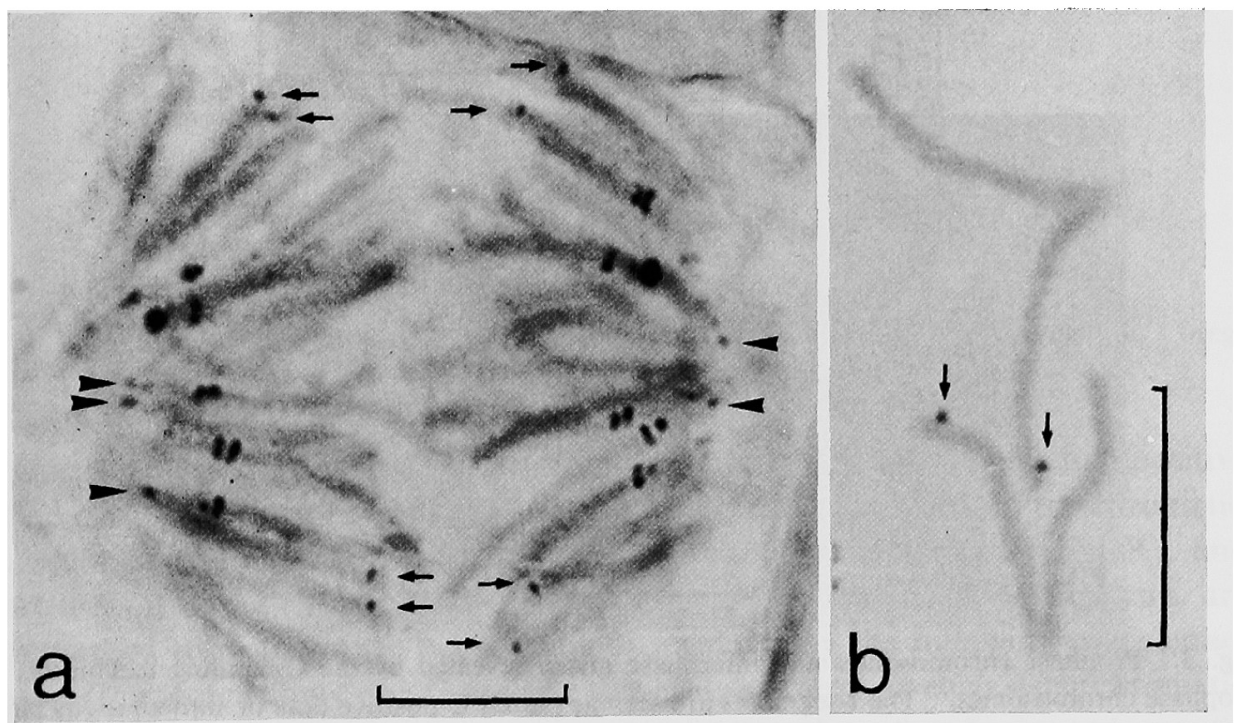

Fig. 2. Anaphase chromosomes revealed by the C-banding technique. a, a representative anaphase plate. The short arms are depicted as single C-positive dots (wedges) and the I-segments are usually composed of two C-positive dots. The four M-segments (arrows) for each haploid chromosome set are visible. b, higher magnification of anaphase chromosomes bearing the M-segments. Each M-segment is situated on the edge of the chromosome. $B a r=10 \mu \mathrm{m}$.

with M-segments, the two C-positive dots were on the outside edge of each chromosome. Rarely, they were located on the inside border of each sister chromatid where they appeared to be one above another (Fig. 1a).

In the anaphase chromosomes (Fig. 2a), most of the I-segments were composed 
of two C-positive dots arranged across the width of the anaphase chromosomes, sister chromatids, but some I-segments appeared as a single C-positive dot (Table 1). This strongly suggests that at metaphase, most of the I-segments are essentially a complex of four C-positive dots arranged in a line across the width of the chromosomes. The S-segments were usually observed as a single C-positive dot which occupied all regions of the short arms (Fig. 2a). The four M-segments of each haploid chromosome set were always seen as a single dot, which was clearly located on the edge of the anaphase chromosome (Fig. 2b).

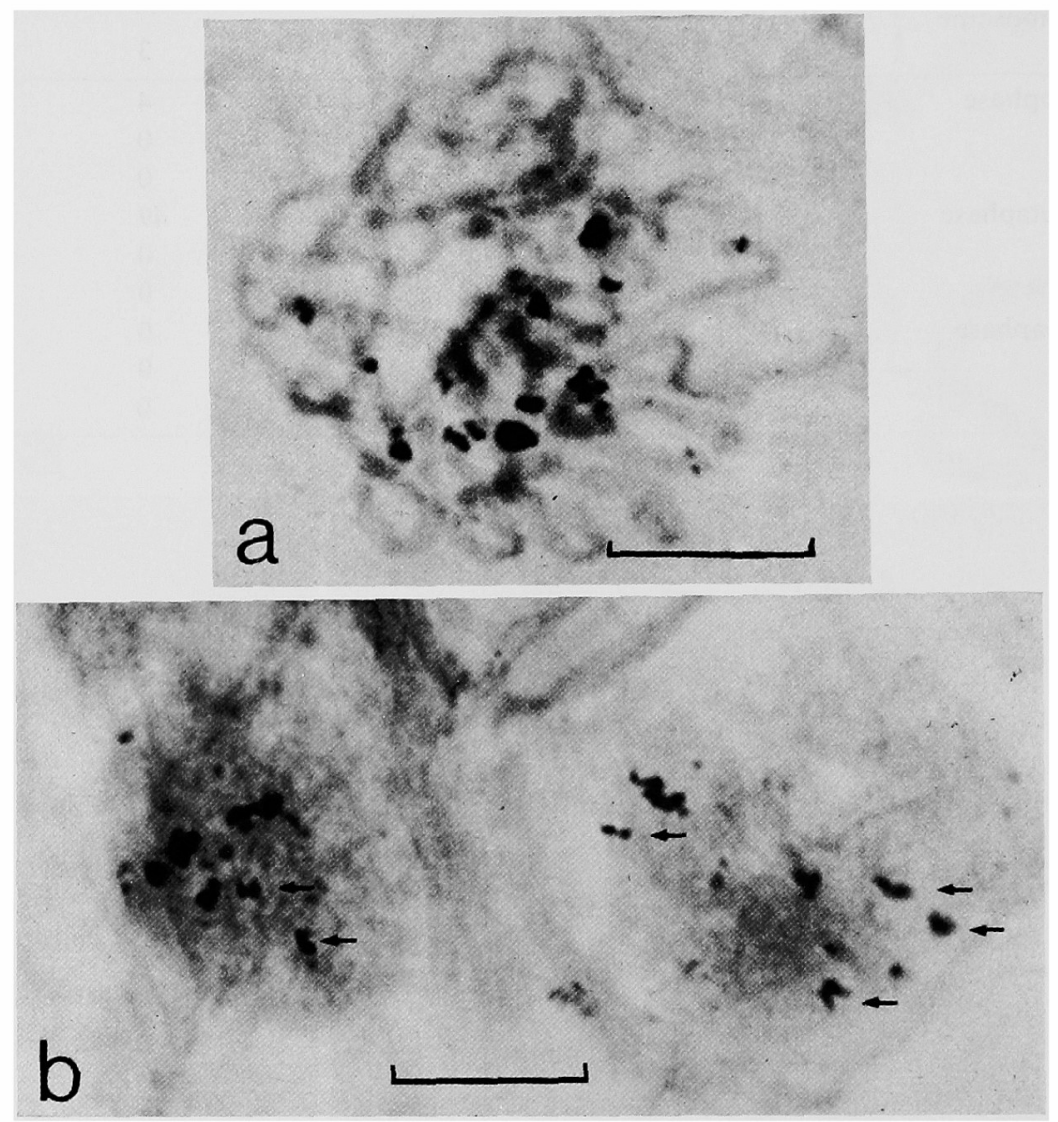

Fig. 3. Prophase chromosomes and interphase nuclei revealed by the C-banding technique. a, prophase chromosomes. The I-segments are seen as paired C-positive dots or sometimes as larger C-positive blocks. b, interphase nuclei. Many paired C-positive dots are still seen (arrows). $\operatorname{Bar}=10 \mu \mathrm{m}$.

At prophase (Fig. 3a), most of the I-segments appeared as paired C-positive dots and those composed of three or four dots were rarely seen (Table 1). The $\mathrm{S}$-segments were observed as single or paired C-positive dots, while the M-segments were always paired dots (Table 1).

In the interphase nuclei (Fig. 3b), paired C-positive dots were frequently observed suggesting that the sister chromatids associate with each other, at least at these regions, during interphase. Large aggregates of C-positive dots were also 
seen. On the whole, the $\mathrm{C}$-positive pattern at interphase was similar to that at prophase.

\section{Discussion}

The I-segments were generally a complex of several C-positive dots except for the I-segments of $\mathrm{S}^{\mathrm{ac}}-3$ chromosomes, which were omitted from the present observations. The fact that the maximum number of C-positive dots per I-segment was four at metaphase and two at anaphase allows the schematic representation of the I-segments as in Fig. 4a. The size of each C-positive dot was less than $1 \mu \mathrm{m}$ and they were arranged closely across the width of the chromosomes, which may easily cause the disappearance and/or fusion of the C-positive dots during the C-banding procedure. The variation in the number of $\mathrm{C}$-positive dots probably depends on their disappearance and/or fusion. This is also supported by the evidence that when the number of the C-positive dots was less than four, one or more of the remaining dots was usually increased in size. In the S-segments, in contrast to the
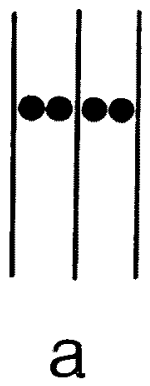
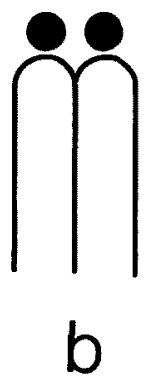
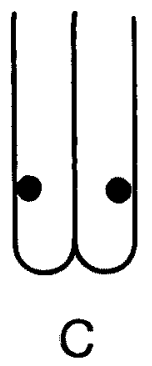

Fig. 4. Schematic representation of the three types of C-banding positive segments. a, I-segment. b, S-segment. c, M-segment.

I-segments, the short arms of the sister chromatids were wholly C-banding positive and no clear differentiation of the C-positive dots across the width of the chromatid was seen (Fig. 4b). They include the nucleolar organizing region (NOR), and

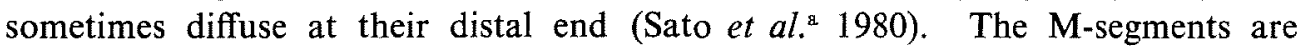
distinguished from the other two groups of segments by the following two characteristics (Fig. 4c): 1) only one C-positive dot is always seen on each sister chromatid and 2) the C-positive dots are always located at the edge of each sister chromatid and they are not as wide as the sister chromatids. At metaphase, these two Cpositive dots were usually situated at the outside of the chromosomes, but they were sometimes at the inside border of two sister chromatids where they were seen one above another. This phenomenon is interpreted as being the result of the chromatid rotary movements that were observed by Bajer (1965).

Especially for the I-segments, the number of the C-positive dots clearly varied depending on the mitotic cell cycle. For instance, the I-segments were composed most frequently of two dots at prophase, three dots at metaphase, and again two dots at anaphase. Two arguments can be presented to explain this phenomenon: 
1) as mentioned above, artificial effects such as the disappearance and/or fusion of the $\mathrm{C}$-positive dots during the $\mathrm{C}$-banding procedure may be involved and 2) differentiation of the $\mathrm{C}$-positive dots actually occurs across the width of the chromosomes during the cell cycle. Considering the facts that, especially at prophase, the chromosomes are very narrow and even though very low in frequency, I-segments composed of four and three C-positive dots are also observed at this stage, the more plausible explanation may be given by the first argument. However, in the Msegments, the two $\mathrm{C}$-positive dots were rarely fused at prophase and metaphase, probably because of their characteristic location.

As seen in the I-segments, the appearance of two C-positive dots arranged across the width of the chromatids seems to support the idea that the chromatid can be subdivided into two subchromatids or half-chromatids (Trosko and Wolff 1965, Wolfe and Martin 1968). However, according to much other data concerning the duplication of chromosomes as revealed by autoradtiographic studies (Taylor $e$ t al. 1957) and by the PFG (fluorescence plus Giemsa) staining technique following treatment with bromodeoxyuridine (Pery and Wolff 1974), it is generally accepted that the chromosomes are unineme. The ultrastructural observations of Comings and Okada (1970) and Comings (1974) support this conclusion. Other studies using the PFG staining technique have demonstrated subchromatid exchanged segments in plant chromosomes (Kihlman 1975, Schvartzman and Cortés 1977, 1978). On the other hand, some authors have reported evidence that the chromatid is a structural unit of chromonema coiling (Kuwada and Nakamura 1934, Ohnuki 1968). Schvartzman and Cortés (1978) argued that, based on chromosome uninemy, each sister subchromatid exchanged segment could be obtained as a result of two exchanges occurring in close proximity in the same coil of chromonema. The characteristic appearance of the C-positive dots, especially in the I-segments and the Msegments, may also be explained by the idea that on the same coil of the chromonema, the chemical and/or structural differentiation of the chromatin occurs.

In a previous study (Sato 1980), three groups of C-banding positive segments could be easily distinguished on the basis of their response to the fluorochromes. It is interesting that the characteristic histochemical nature of the three groups of $\mathrm{C}$-banding positive segments corresponds to the different organization of the Cpositive dots.

\section{Summary}

In the chromosomes of Nothoscordum fragrans, the C-banding positive segments were found to be composed of dot-like compartments (C-positive dots). Three groups of C-banding positive segments could be distinguished by the organization of the C-positive dots: the I-segments were generally composed of four C-positive dots, which were linearly arranged across the width of the metaphase chromosomes, while the S-segments of each sister chromatid were completely C-banding positive and generally appeared as double C-positive dots at metaphase and a single dot at anaphase, and the $\mathrm{M}$-segments of the metaphase chromosomes were almost always composed of two C-positive dots each of which was located on the edge of a 
sister chromatid. The number of C-positive dots for each C-banding positive segment varied depending on the cell cycle. The differentiation of the C-positive dots across the width of the chromosomes is discussed with respect to the chromosomal structure.

\section{Acknowledgement}

The author is thankfull to Dr. C. A. Brown, School of Medicine, University of Hawaii, for correcting the grammatical errors of this manuscript.

\section{References}

Bajer, A. 1965. Subchromatic structure of chromosomes in the living state. Chromosoma (Berl.) 17: 291-302.

Comings, D. E. 1974. The structure of human chromosomes In: The Cell Nucleus, Vol. 1 (Busch, H., ed.), pp. 537-563. Academic Press, New York and London.

- and Okada, T. A. 1970. Do half-chromatids exist? Cytogenetics 9:450-459.

Kihlman, B. A. 1975. Sister chromatid exchanges in Vicia faba II. Effects of thiotepa, caffeine and 8-ethoxycaffeine on the frequency of SCE's. Chromosoma (Berl.) 51: 11-18.

Kuwada, Y. and Nakamura, T. 1934. Behaviour of chromonemata in mitosis II. Artificial unravelling of coiled chromonemata. Cytologia 5: 244-247.

Ohnuki, Y. 1968. Structure of chromosomes I. Morphological studies of the spiral structure of human somatic chromosomes. Chromosoma (Berl.) 25: 402-428.

Perry, P. and Wolff, S. 1974. New Giemsa method for the differential staining of sister chromatids. Nature (Lond.) 251 : 156-158.

Sato, S. 1980. Differential staining of C-bands with acridine orange and Hoechst 33258 in chromosomes of Nothoscordum fragrans. Cytologia 45: 127-136.

-, Ohta, S. and Kuroki, Y. 1980. Heteromorphic appearence of acrocentric nucleolus organizer regions in Nothoscordum fragrans. Cytologia 45: 87-96.

-, Kuroki, Y. and Ohta, S. 1979. Two types of color-differentiated C-banding positive segments in chromosomes of Nothoscordum fragrans, Liliaceae. Cytologia 44: 715-725.

Schvartzman, J. B. and Cortés, F. 1977. Sister chromatid exchanges in Allium cepa. Chromosoma (Berl.) 62: 119-131.

-, - and López-Sáez, J. F. 1978. Sister subchromatid exchanged segments and chromosome structure. Exp. Cell Res. 114: 443-446.

Taylor, J. H., Woods, P. S. and Hughes, W. L. 1957. The organization and duplication of chromosomes as revealed by autoradiographic studies using tritium-labelled thymidine. Proc. nat. Acad. Sci. (Wash.) 43: 122-128.

Trosko, J. E. and Wolff, S. 1965. Strandedness of Vicia faba chromosomes as revealed by enzyme digestion studies. J. Cell Biol. 26: 125-135.

Wolfe, S. L. and Martin, P. G. 1968. The ultrastructure and strandedness of chromosomes from two species of Vicia. Exp. Cell Res. 50: 140-150. 